\title{
La situación de la enfermería en el mundo y la Región de las Américas en
}

\section{tiempos de la pandemia de COVID-19}

Forma de citar Cassiani SHB, Munar Jimenez EF, Umpiérrez Ferreira A, Peduzzi M, Leija Hernández C. La situación de la enfermería en el mundo y la Región de las Américas en tiempos de la pandemia de COVID-19. Rev Panam Salud Publica. 2020;44:e64. https://doi. org/10.26633/RPSP.2020.64

\section{Sr. Editor:}

El informe Situación de la enfermería en el mundo 2020: invertir en educación, empleo y liderazgo (1), lanzado por la Organización Mundial de la Salud (OMS) el 7 de abril de 2020 proporciona una visión y una agenda prospectivas para las políticas relacionadas con esta profesión, al tiempo que el mundo celebra en 2020 el Año Internacional de los Profesionales de Enfermería y Partería y enfrenta la pandemia de enfermedad por coronavirus 2019 (COVID-19).

En el mundo hay aproximadamente 28 millones de profesionales de enfermería, 30\% de los cuales, o unos 8,4 millones, trabajan en la Región de las Américas. Estos profesionales representan el $56 \%$ de la fuerza laboral de salud total (constituida por dentistas, enfermeros, farmacéuticos, médicos y parteras), un porcentaje inferior al promedio mundial, estimado en $59 \%$. Más del $80 \%$ de los profesionales de enfermería se encuentra en países cuyas poblaciones, en conjunto, representan la mitad de la población mundial. A nivel mundial se estima una escasez de 5,9 millones de profesionales, y se calcula que el 89\% (5,3 millones) de este déficit se concentra en los países de ingresos bajos y medianos-bajos.

En la Región de las Américas, el 59\% de esta fuerza de trabajo corresponde a enfermeros profesionales y el $37 \%$ a profesionales asociados; a nivel mundial, en cambio, hay un $69 \%$ de enfermeros profesionales y un $22 \%$ de profesionales asociados. Es importante destacar que en el informe se utilizó la terminología definida por la Clasificación internacional uniforme de ocupaciones de 2008 para reportar informaciones comparables entre los países, y se observa también el elevado número de términos utilizados para denominar a los profesionales de enfermería en los países y la ausencia de consenso de lo que es ser enfermero y su perfil profesional.

A nivel mundial, la fuerza laboral de enfermería es relativamente joven: el 38\% de los profesionales tiene menos de 35 años, en comparación con el 17\%, de 55 años o más. En relación con la distribución etaria existe asimismo un reto mayor, representado por los profesionales que se encuentran cercanos a la edad de jubilación. Se estima que uno de cada seis profesionales de enfermería en el mundo se jubilará en los proximos diez años, y la Región de las Américas es la región de la OMS con el mayor porcentaje de personal de enfermería de 55 años o más (alrededor del 24\%).

Con respecto a la formación profesional, en la Región de las Américas los cursos de grado tienen una duración de 3 años en la mayoría de los países del Caribe, Ecuador y Venezuela; 4 años en Belice, Brasil, Jamaica, México, Panamá, Paraguay, República Dominicana, Trinidad y Tobago y Uruguay; y 5 años en Bolivia, Cuba, Chile, Colombia, El Salvador, Guatemala, Honduras y Perú. A nivel mundial, la mayoría de los cursos son de 3 años de duración.

También según el informe, la Región produce 81,2 graduados en enfermería por 100000 habitantes cada año. Este es el número más alto de todas las regiones de la OMS, con 10 veces más graduados que las regiones de África y el Mediterráneo Oriental. El informe también menciona la existencia amplia de mecanismos regulatorios de las condiciones de trabajo y de la formación de profesionales. El 64\% de los países utiliza un examen de licencia para evaluar y hacer cumplir un nivel mínimo de conocimiento inicial o "aptitud para la práctica" de los profesionales de enfermería graduados antes de acreditarlos para el ejercicio profesional. Sin embargo, las Américas también es la región de la OMS con estándares más bajos para la educación interprofesional (49\%), lo que evidencia la necesidad de avanzar más en este ámbito, ya que los profesionales de enfermería representan más de la mitad de los trabajadores de salud y desarrollan tanto acciones específicas de cuidado de enfermería como acciones complementarias a las demás áreas de la salud.

En muchos países del mundo existe una tendencia a implementar la formación de "enfermeros de práctica avanzada" (EPA). El fortalecimiento de las funciones más avanzadas de enfermería permite, sobre todo, mejorar el acceso y la calidad de la atención en salud, así como aumentar las perspectivas de la carrera y retener a los profesionales en su país. El 55\% de los países de la Región informaron la existencia de funciones atribuibles a profesionales de EPA, aunque algunos países siguen con dificultades para implementar este rol. En América Latina, por ejemplo, no existen ni la regulación ni la formación necesarias para las EPA en la atención primaria de la salud. En el Caribe, los países que más han desarrollado programas con la formación, la regulación y el mercado laboral instituidos son Jamaica y Puerto Rico. Las iniciativas de la Organización Panamericana de la Salud y la OMS han apoyado ampliamente la implementación de enfermeros de práctica avanzada en América Latina y el Caribe (2).

A nivel mundial, en el $37 \%$ de los países se han informado medidas para prevenir ataques a los trabajadores de la salud.

Este es un artículo de acceso abierto distribuido bajo los términos de la licencia Creative Commons Attribution-NonCommercial-NoDerivs 3.0 IGO, que permite su uso, distribución y reproducción en cualquier medio, siempre que el trabajo original se cite de la manera adecuada. No se permiten modificaciones a los artículos ni su uso comercial. Al reproducir un artículo no debe haber ningún indicio de que la OPS o el artículo avalan a una organización o un producto específico. El uso del logo de la OPS no está permitido. Esta leyenda debe conservarse, junto con la URL original del artículo. 
Entre el 1 de enero de 2019 y el 1 de enero del 2020, el Sistema de Vigilancia de Ataques a la Atención de la Salud de la OMS registró 1005 ataques al personal de atención sanitaria, lo que resultó en 198 muertes y 626 lesiones de trabajadores de la salud y pacientes en 11 países que enfrentan emergencias complejas. Esto es particularmente importante en el contexto de la pandemia actual de COVID-19, ya que en los últimos meses han surgido muchos reportes de ataques a profesionales de enfermería y de salud.

Por otra parte, la pandemia de COVID-19 ha dejado al descubierto la vulnerabilidad de los sistemas de salud, así como el déficit de profesionales de enfermería para enfrentar la situación desde la primera línea de atención. Para contener y mitigar la COVID-19 en la Región de las Américas es fundamental contar con equipos de protección individual idóneos y suficientes, acciones de formación continua, condiciones laborales dignas, protocolos acordes a los estándares internacionales, equipos multidisciplinarios e interdisciplinarios, herramientas tecnológicas en el marco de la telesalud y voluntad política desde las instancias directivas de las instituciones de salud. Si se tienen en cuenta y se implementan las medidas anteriores, los profesionales de enfermería tendrán la oportunidad de demostrar todo su potencial como gestores del cuidado mediante acciones de educación y orientación, implementación de prácticas de autocuidado, y comunicación asertiva dirigida a la persona, la familia y la comunidad. El apoyo que puede brindar el personal de enfermería en este escenario es fundamental para mitigar las dificultades que presentan los servicios de salud, así como para favorecer las medidas de protección personal y la abogacía en beneficio del ser humano.

Los datos presentados en el informe exponen desafíos que los países deben considerar en las políticas futuras. Los gobiernos, con el apoyo de otros actores interesados, deben catalizar y liderar una aceleración de esfuerzos para desarrollar la capacidad de liderazgo, administración y gestión de enfermería; avanzar en las agendas relevantes de educación, salud, empleo y género; optimizar el retorno de las inversiones actuales en enfermería mediante la adopción de las opciones de políticas requeridas en educación, trabajo decente, despliegue, práctica, productividad, regulación y retención de la fuerza aboral de enfermería; generar una inversión masiva en la fuerza laboral de la salud y en la enfermería como parte de ella, y aprovechar a la profesión para obtener múltiples resultados de desarrollo, inclusive la creación de empleo, el equilibrio de género y el empoderamiento de los jóvenes.

Para la Región de las Américas, en particular, el informe mundial mostró la necesidad de mejorar la composición de la fuerza de trabajo en enfermería, ampliando el número de profesionales; distribuir mejor los profesionales por países para reducir las grandes diferencias actuales; aumentar la presencia masculina en la profesión; incrementar la dotación de profesionales en las zonas rurales y de difícil acceso; discutir la opción por el examen práctico para aquellos que terminan su formación profesional; e invertir en la formación y el empleo de profesionales de enfermería de práctica avanzada en los países de Amérca Latina. La traducción de la evidencia del informe, las opciones de política recomendada y concreta, las direcciones estratégicas y las decisiones de inversión requerirán coordinación entre los sectores gubernamentales y colaboración con las partes interesadas más críticas. Estos mecanismos de diálogo de políticas deben aprovecharse para obtener las decisiones necesarias en términos de la adopción de políticas sólidas y basadas en evidencia y niveles de inversión apropiados.

La pandemia actual por COVID-19 ha mostrado la necesidad e importancia de disponer de profesionales de salud en número adecuado a las necesidades de cuidados y con buenas condiciones de trabajo (inclusive equipos de protección individual y otros recursos, apoyo al trabajo en equipo y educación continuada), así como el rol relevante que desempeñan los profesionales de enfermería en los sistemas de salud. Los profesionales de enfermería son la primera línea de atención y cuidados y tienen un rol crucial en la mejora del acceso y de la calidad de la atención de salud. El escenario actual puede tornarse especialmente fértil para que los países inviertan en mejorar las condiciones de trabajo y educación en enfermería, lo cual redundará en importantes logros para la cobertura universal de salud y el acceso a la salud de las poblaciones de la Región.

\section{Conflicto de intereses. Ninguno declarado.}

Declaración. Los autores son los únicos responsables de las opiniones expresadas en el manuscrito, que no representan necesariamente la opinión o las políticas de la RPSP/PAJPH o la Organización Panamericana de la Salud (OPS).

\section{Silvia Helena De Bortoli Cassiani}

Organización Panamericana de la Salud/Organización Mundial de la Salud, Washington, D.C., Estados Unidos de América 凶assianis@paho.org

\section{Edgar Fernando Munar Jimenez}

Ministerio de Salud y Protección Social, Bogotá, Colombia

\section{Augusto Umpiérrez Ferreira}

Universidad Católica del Uruguay, Montevideo, Uruguay

\section{Marina Peduzzi}

Universidade de São Paulo, São Paulo, Brasil

\section{Claudia Leija Hernández}

Secretaría de Salud, Ciudad de México, México

Manuscrito recibido el 5 de mayo de 2020. Aceptado para publicación el 6 de mayo de 2020. Sin arbitraje externo.

\section{REFERENCIAS}

1. World Health Organization. State of the world's nursing 2020: investing in education, jobs and leadership. Geneva: WHO; 2020.
2. Organización Panamericana de la Salud. Ampliación del rol de las enfermeras y enfermeros en la atención primaria de salud. Washington, D.C.: OPS; 2018. 